UCRL-ID-132691

\title{
Response to FCC 98-208 Notice of Inquiry in the Matter of Revision of Part 15 of the Commission's Rules Regarding Ultra-Wideband Transmission Systems
}

\author{
Rexford M. Morey
}

December 8, 1998






\section{DISCLAIMER}

This document was prepared as an account of work sponsored by an agency of the United States Government. Neither the United States Government nor the University of California nor any of their employees, makes any warranty, express or implied, or assumes any legal liability or responsibility for the accuracy, completeness, or usefulness of any information, apparatus, product, or process disclosed, or represents that its use would not infringe privately owned rights. Reference herein to any specific commercial product, process, or service by trade name, trademark, manufacturer, or otherwise, does not necessarily constitute or imply its endorsement, recommendation, or favoring by the United States Government or the University of California. The views and opinions of authors expressed herein do not necessarily state or reflect those of the United States Government or the University of California, and shall not be used for advertising or product endorsement purposes.

This report has been reproduced directly from the best available copy.

Available to DOE and DOE contractors from the Office of Scientific and Technical Information

P.O. Box 62, Oak Ridge, TN 37831

Prices available from (615) 576-8401, FTS 626-8401

Available to the public from the

National Technical Information Service

U.S. Department of Commerce

5285 Port Royal Rd.

Springfield, VA 22161 


\title{
Response to FCC 98-208 Notice of Inquiry in the Matter of Revision of Part 15 of the Commission's Rules Regarding Ultra- Wideband Transmission Systems
}

\author{
U.S. Department of Energy \\ Lawrence Livermore National Laboratory \\ P. O. Box 808 \\ Livermore, California 94551-9989 \\ POC: Rexford M. Morey, L-395 \\ Micropower Impulse Radar Program \\ (925) 424-2904 \\ email: morey2@llnl.gov
}

\section{Background}

Lawrence Livermore National Laboratory (LLNL) is a government research and development facility operated by the University of California under contract to the US Department of Energy. The Micropower Impulse Radar (MIR) project was started in the Laser Programs Directorate at LLNL as an outgrowth of the Inertial Confinement Fusion program. New technologies developed and patented by LLNL were directed toward new government applications and also transferred to private industry through commercial licensing agreements. As a laboratory program, MIR operates under DOE contract (Contract 48) with the University of California for performing science and technology work in the national interest. All work performed for external government agencies (DOT, DOD, DOE) have been through "Work For Others" agreements monitored by DOE and the appropriate regulatory agencies. MIR has been licensed to 26 commercial companies for such applications as liquid level measurement, very sensitive motion sensing, and through-the-wall imaging.

In general, Micropower Impulse Radar depends on Ultra-Wideband (UWB) transmission systems. UWB technology can supply innovative new systems and products that have an obvious value for radar and communications uses. Important applications include bridge-deck inspection systems, ground penetrating radar, mine detection, and precise distance resolution for such things as liquid level measurement. Most of these UWB inspection and measurement methods have some unique qualities, which need to be pursued. Therefore, in considering changes to Part 15 the FCC needs to take into account the unique features of UWB technology.

\section{Discussion}

MIR is applicable to two general types of UWB systems: radar systems and communications systems. Currently LLNL and its licensees are focusing on radar or radar type systems. LLNL is evaluating MIR for specialized communication systems. 
MIR is a relatively low power technology. Therefore, MIR systems seem to have a low potential for causing harmful interference to other users of the spectrum since the transmitted signal is spread over a wide bandwidth, which results in a relatively low spectral power density.

The following sections refer to the numbered sections and unnumbered questions in the Notice of Inquiry.

\section{A. What types of UWB devices can we expect to be developed?}

LLNL is performing research and development on short-range non-contacting radar for use in inspection of highway infrastructure, such as bridge decks. These UWB systems are considered ground probing radar (GPR) systems. UWB signals are necessary for imaging the internal structure of highway facilities. No other technology that we are aware of is available today that can provide 3-dimensional images of the internal condition of concrete structures. Other applications include the detection and imaging of land mines, especially plastic mines. Other UWB devices include radar-based liquid level sensors, very sensitive short-range motion detectors/alarms, automotive backupwarning and air-bag sensors, industrial automation sensors, stud finders, very short-range speech monitoring sensors, and medical imaging devices.

\section{B. What are the frequency ranges and bandwidths expected to be used by UWB devices?}

In order for the FCC to effectively regulate UWB devices there must be a useful and concise definition of what constitutes an ultra-wideband emission; in particular a definition of "bandwidth" and "fractional bandwidth". The following definitions are taken from the report ${ }^{1}$ :

(a) Energy bandwidth is the frequency range within which some specified fraction, say 90 or 99 percent, of the total signal energy lies. This may be defined for a single pulse, if all pulses are the same, or for a group of pulses that are processed together to yield a single decision. The upper limit of this range is denoted by $f_{h}$ and the lower limit by $\mathrm{f}_{1}$.

(b) Fractional bandwidth $=2\left(f_{h}-f_{1}\right) /\left(f_{h}+f_{l}\right)$. Note that this definition avoids requiring a definition of the center frequency. However, a center frequency is often estimated and quoted by practitioners as $\left.\left[f_{h}+f_{1}\right) / 2\right]$.

(c) Ultra-wideband emissions are any emissions whose fractional bandwidth is greater than 0.25 regardless of the center frequency or the signal time-bandwidth product.

(d) The choice of 0.25 as the defining value is largely arbitrary, but does in some sense represent the demarcation between conventional narrow-band (NB) and wide-band (WB) techniques.

1 "Assessment of Ultra-Wideband (UWB) Technology", prepared by OSD/DARPA Ultra-Wideband Radar Review Panel, Battelle Tactical Technology

Center, Contract No. DAAH01-88-C-0131, ARPA Order 6049, July 13, 1990. 
Footnote 9 of the Notice of Inquiry presents a useful way to calculate pulse rise time, pulse width and signal bandwidth. A method needs to be established for measuring the bandwidth of UWB signals.

Practitioners will need to establish that UWB devices need to transmit UWB signals to function properly and that emissions across restricted bands do exist. For example systems for imaging inside solid materials, such as bridge decks, will only work with UWB signals. There must be a methodology for showing the requirement for UWB in order to achieve the requisite image resolution, processing gain, range resolution, etc. at a bandwidth and frequency for the application.

The central frequency of an UWB sensor should be selected based on the specific measurement or imaging application. There is no one central frequency that is optimum for all applications. Parameters that influence the optimal frequency in any given application include the properties of the materials to be measured, the presence and properties of any secondary layers, and the presence and properties of particles in the gas space between the materials being sensed and the antenna. It is expected that the center frequency of UWB devices will fall between $20 \mathrm{MHz}$ and $60 \mathrm{GHz}$.

Bandwidth is determined largely by pulse rise-time and pulse-width, which in turn is driven by the need for highly accuracy measurements and for resolving closely spaced targets. Expected pulse rise times will vary from 10 picoseconds (ps) to 5 nanoseconds (ns) and pulse widths may range from $50 \mathrm{ps}$ to $20 \mathrm{~ns}$. Therefore, bandwidth will range from $150 \mathrm{MHz}$ to $30 \mathrm{GHz}$.

\section{C. What are the expected total power levels and spectral power densities, peak and average, of UWB devices?}

Peak power levels are determined primarily by the maximum operating range of the UWB devices and by the reflection efficiency of the target. Maximum operating ranges for MIR devices are expected to be from 5 centimeters for very low power units to 50 meters for deep penetrating units for geophysical applications. Target reflection efficiency depends primarily on target conductivity, dielectric constant and surface smoothness. It is expected that peak transmitter power for most typical MIR applications will be $+20 \mathrm{dBm}$, while medium range MIR devices can be $+40 \mathrm{dBm}$.

Peak spectral power density is expected to be in the range of about $160 \mu \mathrm{W} / \mathrm{MHz}$ (+20 dBm, $500 \mathrm{MHz}$ bandwidth) assuming uniform power distribution within the bandwidth. Spectral power density will be only slightly dependent on bandwidth since the required power increases roughly linearly with the receiver and transmitter bandwidth.

The expected pulse duty cycle might be in the range of about $10^{-4}$ (pulse-width $=1 \mathrm{~ns}$, $\mathrm{PRF}=100 \mathrm{kHz}$ ) depending on the application and required pulse width.

9D. What are the expected or desired operating distances?

Maximum operating ranges for MIR UWB devices are expected to run from $5 \mathrm{~cm}$ for low cost, low power devices to 1000 meters for high power, long range motion sensors.

10A. Are there certain types of UWB devices or applications that should be regulated on a licensed basis under some other rule part? If so, which rule parts? 
LLNL accepts the basic premise for the licensing of low-power UWB devices for imaging, which is that the operator must accept whatever interference is received and must correct whatever interference is caused, even including termination of operation of the device or system, if necessary. (See Paragraph 10.10 "Ultra-Wideband Radar", National Telecommunications and Information Administration (NTIA) Manual.) LLNL thinks that the potential for low power UWB sensors to interfere with other, non-Part 15 devices is very low. This is because of the low spectral energy density and low radiated power of the UWB sensors and the relatively large distances between the sensors and devices under the control of others.

10B. If provisions are made for UWB technology under Part 15, how should we define UWB technology?

A technical definition of UWB technology should focus on the definitions given above for bandwidth, average and peak power, and the particular application that can not be satisfied by existing Part 15 technology. The regulations should be modified as discussed below so as to allow UWB and any other as yet undefined technologies to operate if the technologies can accept the limitations on generating and receiving interference, which form the basis for the existence of Part 15.

11A. Should the rules generally continue to prohibit operation of UWB systems within the restricted bands and the TV broadcast bands?

Part 15 should be modified so as to remove the distinction between intentional radiation and unintentional/spurious emissions in the restricted and TV bands. The purpose of the restricted bands is to prevent interference with certain sensitive and safety related operations. This purpose can be best met by restricting the allowable radiation in a restricted band without regard to whether the radiation is intentional or unintentional/spurious. After all, intentional radiation in a restricted band is no more likely to cause interference to a sensitive or safety related operation than is a spurious signal of the same amplitude in the same band. This simple change would allow UWB sensors to operate in the restricted bands at spectral power levels equal to or below the spurious levels now allowed without causing interference to the sensitive or safety related operations.

11B. Are there certain restricted bands where operation could be permitted, but not others? If so which bands and what is the justification?

Based on our research, LLNL has not found any alternative to permitting intentional radiation in the restricted bands, provided that the levels do not exceed those now allowed for spurious radiation.

11C. If certain restricted bands were retained, what impact would this have on the viability of UWB technology?

That would depend on the number and placement of the restricted bands. The center frequency and bandwidth of an UWB sensor will be limited by the size of the windows between the restricted bands. This limit on bandwidth translates directly from a limit on pulse width and pulse rise time (as discussed in footnote 9 of the Notice of Inquiry), to a limit on the maximum accuracy and clutter rejection performance of the sensor. The use 
of "notch filters" between the UWB transmitter and sensor/antenna in order to remove energy at the restricted bands will cause excessive ringing in the signal applied to the UWB sensor. These limitations and "engineering solutions" will restrict the applicability of UWB technology. See the attachment.

12A. Are the existing general emission limits sufficient to protect other users of the spectrum, especially radio operations in the restricted bands, from harmful interference?

No response.

12B. Should different limits be applied to UWB systems?

The minimum limitations should be applied that are sufficient to protect the licensed users and operations in the restricted bands. These limitations should be sufficiently well defined so as to be applicable to all transmitter types, including UWB, without specifically referring to any transmitter type, including UWB.

12C. Should we specify a different standard for UWB devices based on spectral power density? Should these standards be designed to ensure that the emissions appear to be broadband noise?

It is desirable to add a standard based on spectral power density if such a standard is required to protect licensed users and operations in the restricted bands. However, all standards should apply to all radiators, not just to UWB systems. Ensuring that emissions appear to be broadband noise is probably not sufficient to protect other users since broadband noise of sufficient amplitude will still disrupt other operations. The broadband noise must still remain below the level that is acceptable to other users.

12D. What is the potential for harmful interference due to the cumulative impact of emissions if there is a large proliferation of UWB devices? Could the cumulative impact result in an unacceptably high increase in the background noise level? Should the Commission limit the proliferation by restricting the types of products or should the rules permit manufacturers to design products for any application as long as the equipment meets the standards?

The impact of harmful interference due to the cumulative effect of a large proliferation of UWB devices is not well documented at this time. Theoretical studies and calculations have indicated that there will be an increase in the background noise level. Worse case analysis indicates that the increase will be unacceptably high. If a multitude of UWB transmitters are about the same distance to a "victim receiver" and if all the transmitters are time-synchronized, then the UWB pulses will be additive at the victim receiver. It's as if the number of synchronized individual UWB devices increases the peak-to-peak amplitude of a single UWB transmitter. This scenario seems to be highly unlikely, but needs to be verified to the satisfaction of other users. This concern applies primarily to a network of UWB communication systems where the intent is to transmit over ranges of 100 to 1000 meters. In LLNL's years of experience with UWB devices, this concern does not seem to be a problem for low power, short-range devices such as motion sensors or for liquid level sensors that are contained in a tank or other metal enclosure. Also, in our opinion, harmful interference will not occur due to the 
cumulative impact of UWB ground penetrating radar systems, which radiate the main lobe into the ground.

Therefore, at this time, the Commission should limit the proliferation of UWB communication devices by restricting the types of communication products, which in their operational mode have emission levels greater than unintentional radiators. If regulation is necessary to limit the cumulative impact of a proliferation of UWB devices on the background noise, two different standards should be written, one for devices used in the home and a more lenient one for industrial use. This would be similar to the regulation of class $\mathrm{A}$ and class $\mathrm{B}$ computing devices.

\section{E. Should a limit on the total peak level apply to UWB devices?}

Yes, there should be a limit on the peak power transmitted by a UWB device. However, that peak should be based on the potential for the UWB pulse to cause harmful interference to existing users of the radio spectrum. Therefore, the peak limit should be a fixed power level and not a multiple of the average transmitted power.

12F. Can emissions below or above a certain frequency range be further filtered to reduce the potential for interference to other users of the radio spectrum without affecting the performance of the UWB systems?

As stated above, imaging systems require their full bandwidth capability to function and, therefore, any filtering, such as notch filtering will adversely affect their performance; this is not only a function of frequency range but of bandwidth. Other UWB devices, such as motion sensors and sensors for accurately measuring distance, can be designed to operate in a certain frequency range with minimal energy in the restricted bands. However, this level of energy in the restricted band can not be zero, as is the interpretation of the present situation for intentional radiators.

12G. Are the existing limits on the amount of energy permitted to be conducted back onto the AC power lines appropriate for UWB devices?

Yes, however, if further limits are necessary for UWB devices used in the home, separate standards for home and industrial environments should be adopted.

\section{H. What operational restrictions, if any, should be required to protect existing users?}

A modified licensing procedure may be needed for UWB imaging systems, such as ground penetrating radar units. This may include requiring notification of time, place and duration of a UWB GPR survey, so that if an existing user experiences harmful interference, the UWB devise can be immediately shut down. (See for example, Paragraph 10.10 "Ultra-Wideband Radar", National Telecommunications and Information Administration (NTIA) Manual.)

12I. Is the use of UWB modulation techniques necessary for certain types of communication systems; if so, for what purposes?

No response. 
13A. Is a pulse desensitization correction factor appropriate for measuring emissions from a UWB device? Should any modifications be made to this measurement procedure for UWB devices?

No, the pulse desensitization correction factor is not appropriate for measuring emissions from a UWB device. The ratio of peak to average power levels in a UWB sensor may be in the range of 100 to 10,000 . This is primarily a function of the duty cycle. Measurement of the peak power level is very difficult with a spectrum analyzer. A measurement technique needs to be developed using a fast sampling oscilloscope or similar device to measure the pulse shape, including pulse rise time, peak-to-peak voltage amplitude, and PRF. Then regulations established to limit the voltage amplitude, the PRF, and the ratio of amplitude to PRF for the UWB transmitter. This testing should be done on UWB devices at one FCC test facility, such as Columbia, MD.

13B. Would another measurement procedure that does not apply a pulse desensitization correction factor be more appropriate for determining the interference potential of an UWB device?

Yes. See the answer to $13 \mathrm{~A}$ above.

13C. The frequency range over which measurements are required to be made depends on the frequency of the fundamental emission. Is the frequency of the fundamental emission readily discernible for UWB devices? Are the current frequency measurement ranges specified in the rules appropriate for UWB devices or should these ranges be modified?

There is no clearly defined fundamental emission frequency for a UWB device, and the concept of a fundamental frequency has no real meaning for a UWB device. Therefore, the current frequency measurement ranges specified in the rules are not appropriate for UWB devices. Rather, the highest frequency, which is generally a function of pulse rise-time, should be used to determine the frequency range over which to make measurements. Frequency range is determined from the pulse rise time, pulse shape, and pulse width and formulas such as those in footnote 9 of the Notice of Inquiry.

13D. Are the measurement detector functions and bandwidths appropriate for UWB devices? Should these standards be modified and, if so, how?

Use of a spectrum analyzer to measure peak and average power levels and bandwidths is difficult or impossible because of the low duty cycle and low spectral energy density. New measurement techniques must be developed based on the use of a fast sampling oscilloscope to make time based measurements and, then calculate the equivalent frequency components and energy densities. These new measurement methods, base on time-domain techniques, should then be combined with the appropriate existing frequency domain methods using a spectrum analyzer.

13E. Are there any other changes to the measurement procedures that should be applied to UWB devices?

To LLNL's knowledge and experience there are none, other than those above. 
14A. Should the prohibition against Class B, damped wave emissions apply to UWB systems or is the prohibition irrelevant, especially in light of the relatively low power levels employed by UWB devices?

The prohibition against Class B, damped wave emissions does not seem to serve any useful purpose at this time and should not be applied to UWB systems. It was apparently intended to prohibit the use of spark gap type transmitters in the early 1900s and is now redundant since the rules regarding spurious emissions also prohibit spark gap transmitters.

14B. Comments are invited on any other matters or issues that may be pertinent to the operation of UWB systems.

LLNL has no other comments.

The following attachment shows the effect of a notch filter on a UWB signal. 
1. Effect of notch filtering the restricted bands

Filter type: elementary LC resonator filter tuned to the restricted bands

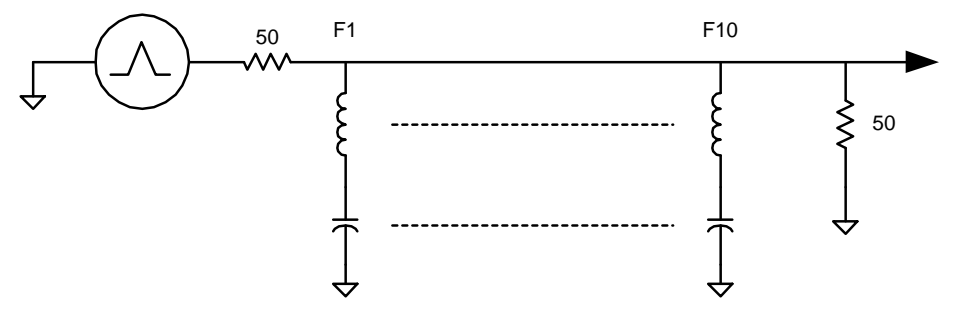

Frequency response

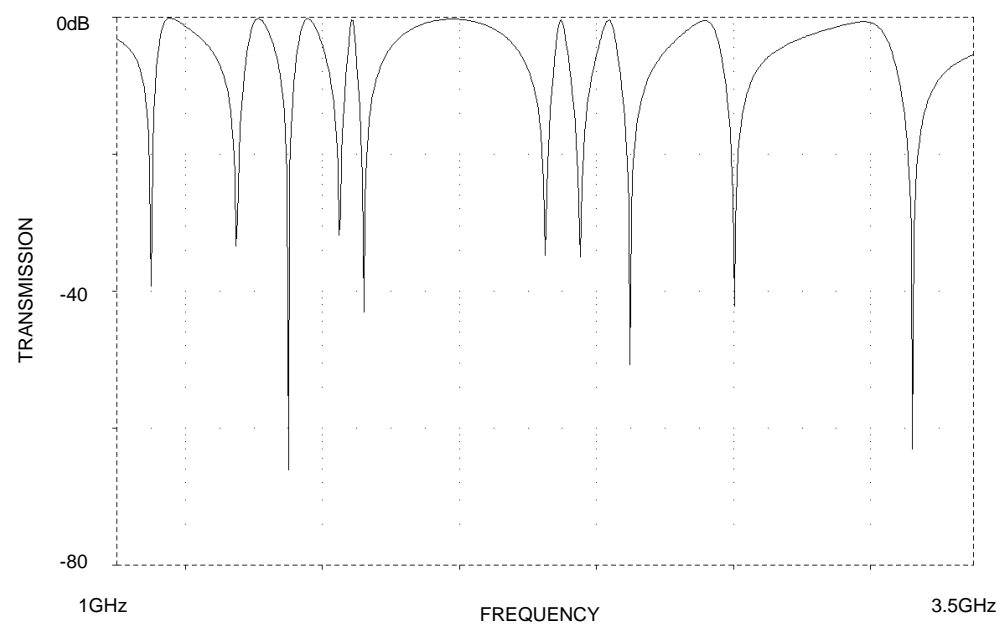


100ps Impulse response

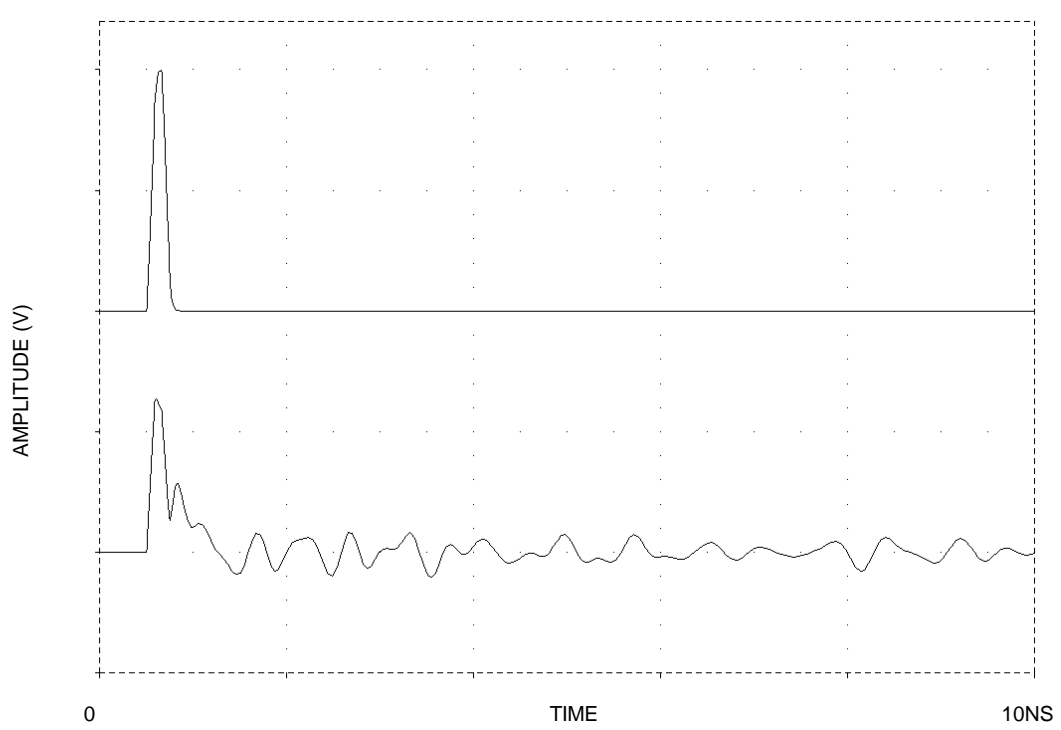

The effect of notch filtering is to convert a UWB signal into an aggregate of narrowband signals. Notch filtering will substantially impair UWB imaging, it stretches main bang ringing so weak returns are blinded, and it effectively eliminates the possibility for timecoded channelization. Depending on the filter $Q$ and the PRF, filter ringing will extend from one impulse to the next and will build in amplitude to a greater extent than the single impulse response shown above. 